\section{Áreas susceptibles de riesgo en localidades de pobreza extrema en el sur de Yucatán}

DOI: $10.22403 /$ UQROOMX/TYP02/04

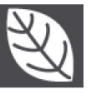

Resumen
Oscar Frausto," Thomas Ihl, Justo Rojas, Steffi Goldacker, Geiser Chale, Steffen Giese, Jobst Wurl, Pilar Careaga y Rubén Bacab

La falta de herramientas para la mitigación de los daños ocasionados por el huracán Isidoro a la península de Yucatán en septiembre de 2002 dio lugar a esta investigación geográfica, cuyo propósito es estudiar los elementos del medio natural que determinan la existencia de áreas de alto riesgo de desastres naturales, las cuales son identificadas mediante indicadores socioeconómicos de localidades del sur de Yucatán. La identificación parte, primero, del mapeo de las formas y elementos del relieve que definen la geomorfología; ésta se determina por el análisis del relieve, enfocando la génesis, el proceso y la forma. Se reconocen así las áreas susceptibles de inundación, diferenciadas en ordinarias, extraordinarias y permanentes. Conjuntamente, se identifican las localidades en pobreza extrema, considerando nueve dimensiones socioeconómicas que se resumen en los índices Necesidades básicas insatisfechas y Línea de pobreza. Finalmente, los resultados de este trabajo se sintetizan en: a) Mapas de riesgo de inundaciones del sur de Yucatán, a escala 1:50 000 y, b) Identificación de las localidades en pobreza extrema susceptibles de inundación.

\begin{tabular}{r|l} 
Palabras & $\begin{array}{l}\text { Yucatán, geomorfología, peligros naturales, pobreza extrema, } \\
\text { CLAVEesgo. }\end{array}$
\end{tabular}

*Universidad de Quintana Roo / ofrausto@uqroo.mx 
Las investigaciones geográficas sobre riesgos naturales pueden aportar parámetros importantes para la evaluación, manejo y planeación del medio donde habita el hombre. El análisis de los fenómenos naturales y tecnológicos que provocan desastres, la vulnerabilidad de la población ante un fenómeno peligroso, y las situaciones de contingencia ante los desastres, así como la función y transformación del territorio, dan por resultado la evaluación del riesgo del lugar.

Las investigaciones geográficas de carácter preventivo (evaluación de la amenaza) permiten identificar el tipo de fenómenos peligrosos que, sumados con la vulnerabilidad humana, inducen al riesgo. El resultado de estos trabajos se remite a la ubicación de lugares de ocurrencia, al tipo de fenómenos que provocan desastres, su génesis, su regularidad, estadios de evolución y ciclos dinámicos, además de su planeación y prevención (Frausto, 2002).

Batista y Sánchez (1995) clasifican los riesgos con base en su origen: huracanes, lluvias intensas, inundaciones, tornados, penetración del mar y marejadas; sismos, maremotos y tsunamis; deslizamientos de tierra, avalanchas y aludes; erupciones volcánicas; sequías intensas; incendios forestales; heladas y granizadas. Esta clasificación se distingue por mostrar fenómenos potencialmente dañinos para zonas intertropicales.

Por otro lado, Hewitt (1997), a raíz de un estudio detallado de las causas y consecuencias de los fenómenos naturales y antrópicos que desencadenan desastres, divide los riesgos en: a) naturales (atmosféricos, hidrológicos, geológicos y biológicos); b) tecnológicos (manejo de sustancias peligrosas, procedimientos peligrosos y medios peligrosos); c) violencia social (medios de violencia, perpetradores y métodos) y, d) complejos (hambruna, crisis de refugiados, derrames tóxicos, pruebas nucleares y explosiones).

El concepto de riesgo es resultado de una continua transformación de las ciencias. En Geografía, las investigaciones sobre riesgo se inician en la década de los años veinte, al identificar los agentes causales de los peligros se incide en la elaboración de políticas que permiten mitigar el impacto de los mismos (Burton, Kates y Snead, 1969).

En la década de los años cuarenta, las investigaciones toman otro rumbo, se centran en el estudio de los procesos físicos de los peligros (Whyte, 1975) y aspectos sociales de percepción (Burton y Kates, 1962), lo que indica la primera división conceptual en los estudios de peligro o amenaza y vulnerabilidad. 
Con el trabajo de Mittchell (1980) se sistematiza la investigación de los procesos de los riesgos, denotando que los estudios de los eventos naturales que representan peligro para la sociedad deben de analizar las características principales del fenómeno, que se reflejan en la magnitud, extensión espacial, localización, ciclos de comportamiento y periodicidad.

Burton (1978) propone siete variables en relación con la descripción del riesgo: I. Magnitud (alta / baja);2.Velocidad de emplazamiento, ambas pueden ser cuantificadas instrumentalmente (pluviómetros o diseños técnicos propios para cada fenómeno) o descritos por inferencias estadísticas; 3. Frecuencia (frecuente / raro); 4. Duración (largo / corto), los cuales se caracterizan por medio de análisis estadísticos o cronológicos; 5 . Espaciamiento temporal (regular / irregular) distinguiendo entre fenómenos en secuencia o al azar (esporádicos); 6. Extensión espacial (limitado / ilimitado) y, 7. Dispersión espacial (difuso / concentrado), que presenta patrones puntuales, lineales o en áreas.

En 1997, Frampton et al. proponen una alternativa para clasificar el riesgo, con un modelo donde la génesis, tipo, agente, intensidad, frecuencia, distribución y niveles de energía son considerados.Además, destaca el dominio del carácter sectorial que ha predominado en los estudios de la amenaza.

Tomando en cuenta este punto de vista de enlazar los fenómenos naturales, que destaca el comportamiento, la dinámica y ciclos de actividad, además de las diferencias económicas y sociales de la población en un análisis de pobreza,' se puede elaborar una cartografía de riesgo. El objetivo es mostrar las regiones donde se presentan fenómenos naturales peligrosos que en un momento dado inducen al riesgo y desastre.

Los estudios de riesgo en México han sido abordados desde una perspectiva analítica, identificando y describiendo los procesos naturales modeladores del paisaje, estos estudios hacen énfasis en aquellos procesos considerados inestables. Ejemplo de lo anterior son los mapas de susceptibilidad a fenómenos naturales que causan desastres en México y la cuenca de México (Ortiz et al., 1996a; Zamorano et al., 1992), el estudio de las zonas minadas de la delegación Álvaro Obregón realizado por Cordero (1992),

'Centrado en el estudio de Necesidades básicas insatisfechas (NBI) y Línea de pobreza (LP) de la localidad, en el cual se destacan los desequilibrios internos del municipio y se toma en cuenta la disparidad intrarregional que permite identificar las localidades de extrema pobreza desde una aproximación de estudios comparados.
89

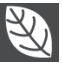


enfocado a los colapsos del terreno; asimismo, el trabajo de García (1994), quien analiza los componentes del medio natural para evaluar el territorio desde una perspectiva geográfica, identificando regiones vulnerables a desastre, es un ejemplo de esta perspectiva. En 1995, Palacio (1995) realiza un estudio de riesgo mediante un análisis geosistémico orientado a la evaluación de los fenómenos naturales peligrosos y a las condiciones socioeconómicas de la población.

En particular, la península de Yucatán ha sido estudiada desde dos perspectivas. Una se orienta a la descripción de los fenómenos hidrometeorológicos (lluvias, tormentas, inundaciones, sequías, erosión y depositación costera -Orellana et al., 1999; Lugo y García, 1999-); en otra, se sintetiza la relación entre los fenómenos naturales y la población, principalmente en mapas de peligros geomorfológicos y geológicos (Ortiz et al., 1997). Finalmente, Oropeza (1999) define los sistemas vulnerables y de impacto ambiental de la península. En estos antecedentes, la región sur de Yucatán resalta por su alta vulnerabilidad y mayor impacto, por fenómenos naturales peligrosos (de tipo geomorfológico y geológico), sin embargo, debido a la escala de trabajo (I:I 000 000, con resolución a I:250 000) no es posible utilizarlos como instrumentos y herramientas para la toma de decisiones, por lo que es imperante el estudio a detalle (escala 1:50 000) del área de riesgo con el fin de definir puntualmente aquellas localidades expuestas a desastres, por sus riesgos y por su pobreza extrema, lo cual es el objetivo de este trabajo.

Yucatán es el estado más poblado de los tres que configuran la península homónima. Su población representa menos de $2 \%$ de la mexicana y su densidad media es inferior al promedio nacional. En términos estadísticos, los indicadores confirman que Yucatán escapa a la situación de grave pobreza que aqueja a las regiones meridionales del país, pero un análisis detallado de estos indicadores, entre ellos la dinámica poblacional y la actividad económica, advierte que la sociedad yucateca afronta perspectivas de desarrollo económico y social en condiciones de extrema precariedad (Córdoba, 1999).

Lo anterior es aplicable a los municipios de Tekax,Tzucacab y Peto (figura I), donde ocho localidades definen la estructura intramunicipal (Tekax, Tzucacab, Peto, Catmis, Becanchen, Kancab, Penkuyut y Xaya) y más de I 30 localidades pequeñas que configuran las relaciones y diferencias microregionales del cono sur. La población total de estos municipios es de 65949 habitantes (en 1995). Los tres municipios se caracterizan por tener una densidad de población 


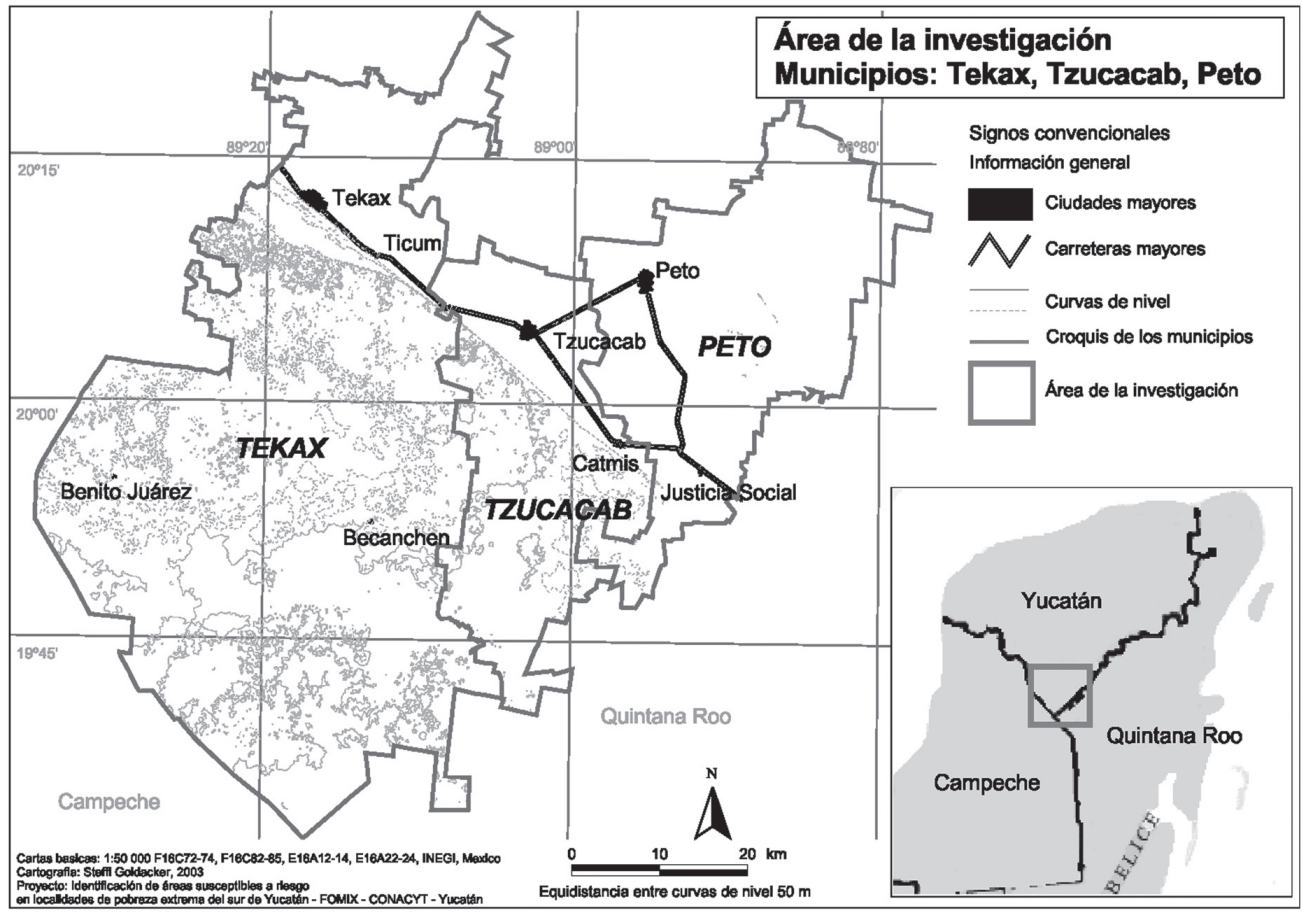

Figura I. Área de estudio en el cono sur de Yucatán

menor a 20 habitantes $/ \mathrm{km}^{2}$, con un poblamiento disperso en localidades pequeñas y muy pequeñas con viviendas aisladas. Asimismo, su población se caracteriza por percibir menos de dos salarios mínimos o sin ingresos (más de $90 \%)$.

Los indicadores de salud no son alentadores, los tres municipios se caracterizan por sus altos niveles de mortandad en niños menores a cinco años (más de $11.4 \%$ ) y por prevalecer un porcentaje mayor a $50 \%$ de desnutrición (Cervera y Méndez, 1999). Las viviendas con agua potable no superan $60 \%$ y más de $40 \%$ de las viviendas tienen piso de tierra. La población sustenta su económica en las actividades primarias. En conjunto, se ubican en la región frutícola. 
Desde el aspecto fisiográfico del territorio, su variedad le imprime una característica peculiar al cono sur (lomeríos y planicies), la precipitación anual oscila de I 000 a I $050 \mathrm{~mm}$, con anomalías de hasta $150 \mathrm{~mm}$. Peligros sanitarios en agua y suelo, y zonas con problemas de cuña salina por la sobre explotación intensiva del agua, colapsos superficiales del suelo, huracanes, tormentas tropicales y nortes e incendios forestales, así como contaminación del agua subsuperficial, son los fenómenos de riesgo y de peligro hasta ahora señalados por los estudios de la región. Sin embargo, su caracterización se hace desde una aproximación del diagnóstico, lo cual evidencia la falta de información precisa para la toma de decisiones y la gestión municipal en caso de desastre.

Lo anterior se puso de manifiesto durante la contingencia del huracán Isidoro en septiembre del 2002, donde el territorio de Yucatán fue impactado por fenómenos hidrometeorológicos asociados al huracán (vientos, inundaciones, contaminación de acuíferos y destrucción de infraestructura carretera y viviendas).

La dispersión de las localidades y la calidad de las vías de comunicación imposibilitaron el auxilio de los afectados; pero el principal obstáculo para la toma de decisiones fue la falta de instrumentos de apoyo, en este caso, mapas a detalle sobre las áreas afectadas y las áreas latentes de riesgo e impacto. Estos instrumentos básicos y de fácil interpretación y manejo permiten la gestión a todos los niveles y pueden ser utilizados por la población para mitigar los efectos en caso de contingencia y riesgo.

La correlación de los aspectos ambientales peligrosos y la vulnerabilidad social permite la identificación de zonas de riesgo, enfocada en este escrito a la susceptibilidad de inundación durante fenómenos naturales extraordinarios; y a la clasificación y localización de comunidades vulnerables por sus condiciones de pobreza extrema. La identificación parte de dos aspectos diferentes. Primero, el mapeo de las formas y elementos del relieve, que definen la geomorfología (figura 2); ésta se determina por el análisis del relieve, enfocando la génesis, el proceso y la forma, se reconocen así aquellas susceptibles de inundación, diferenciadas en ordinarias, extraordinarias y permanentes. Conjuntamente, se identifican las localidades en pobreza extrema, tomando en consideración nueve dimensiones socioeconómicas, que se resumen en dos índices (Necesidades básicas insatisfechas y Línea de pobreza). Esta es la hipótesis de la investigación cuyo objetivo se sintetiza en 
la presentación del Mapa de riesgo a inundaciones del sur de Yucatán a escala I:50 000 y la Identificación de las localidades en pobreza extrema susceptibles de inundaciones.

\section{Materiales y métodos}

En la realización de este trabajo se siguieron cuatro métodos principales.

\section{I.Análisis ambiental}

a) Con el fin de identificar la frecuencia y los ciclos de recurrencia de fenómenos hidrometeorológicos con características de desastre, se realizó una búsqueda de información en crónicas y archivos históricos. Se identifica la recurrencia, magnitud e intensidad de cada una de las inundaciones.

b) Análisis sistémicos de la geología, edafología, hidrología y clima. En la primera es posible señalar los elementos determinantes de la estructura del relieve, así como los componentes del suelo que permitieron inducir la predisposición del territorio a ciertos fenómenos (inundaciones, agrietamiento del suelo, deficiencias en el drenaje, colapsos superficiales, entre otros), La información hidrológica y climática nos auxilió en el planteamiento de ciclos de recurrencia, frecuencia e intensidad de inundaciones, sequías y lluvias torrenciales.

c) El análisis de la información sobre precipitación (obtenida de las estaciones climáticas pertenecientes al Servicio Meteorológico Nacional y la Comisión Nacional del Agua) para el periodo I92 I-2002 nos ha permitido reconocer periodos de lluvia y sequía, y magnitud y frecuencia de las inundaciones.

d) Elaboración del sistema de información geográfica del relieve y de las dimensiones socioeconómicas de las localidades.

e) Se realizaron mapas morfométricos que permitieron la elaboración del modelo geomorfológico del sur de Yucatán, destacan la identificación y caracterización de los fenómenos naturales peligrosos y la susceptibilidad del territorio a riesgo y desastre; comprenden: 
a) altimetría; b) inclinación del terreno; c) modelos de elevación (MDT);

d) red fluvial y, e) carta de densidad y profundidad de la disección.

f) Fotointerpretación. Facilita la interpretación de las fotografías aéreas y su representación cartográfica. Se utilizaron dos escalas en la interpretación de las fotografías aéreas: I: 75 000; 1999 y I:19 000; 2000. A partir de esta interpretación se detectarán regiones donde la dinámica representa susceptibilidad de inundaciones para las localidades.

2.Análisis social

A partir del análisis de las condicionantes sociales y económicas que definen la pobreza (Boltvinik y Hernández, 1999), se determinaron los patrones de distribución de pobreza y fueron identificadas las localidades en pobreza extrema. Se consideraron seis dimensiones para diferenciar la pobreza: a) inadecuación de la calidad y cantidad de la vivienda; b) inadecuación de las condiciones sanitarias; c) inadecuación de otros servicios; d) inadecuación del patrimonio básico; e) rezago educativo; f) inadecuación de acceso a la atención de salud y a la seguridad social $y, g$ ) inadecuación al empleo. Nuestro indicador básico fue la localidad. Para la identificación de la población pobre y la no pobre en cada una de las dimensiones y de manera integrada, se procedió a: I. Clasificar la población y localidades con el fin de identificar aquellos en pobreza extrema; 2 . Se caracterizó a la población y las localidades según su incidencia, intensidad y magnitud de pobreza $y$, 3. Se presentó un perfil de la población en pobreza extrema.

\section{Trabajo de campo}

Mediante recorridos de verificación y corrección de fronteras fue posible detallar y enriquecer la descripción de manera puntual, que debido a la escala de trabajo no había sido posible obtener por métodos indirectos. Conjuntamente, se revisó la información vertida en el análisis de pobreza para localidades clave (se levantaron más de 2000 encuestas sobre el NBI y el LP), además de la referente a la comprobación de datos y la descripción de actitudes antes, durante 
y después del huracán Isidoro, asimismo, se registró la forma en que la población se ha adaptado después del desastre, para definir la resiliencia de las localidades ante estos fenómenos.

4. Integración de la información

La identificación de las áreas susceptibles de riesgo de inundaciones se hizo a partir de una sobreposición cartográfica temática a través del uso de un Sistema de Información Geográfica (SIG), todo generado a escala 1:50 000 y a nivel de localidad. El análisis ambiental y el análisis social fundamentan la cartografía base para la sobreposición. Finalmente, se obtuvo el padrón de localidades en pobreza extrema y áreas susceptibles de inundaciones.

\section{Resultados}

Áreas susceptibles de inundaciones

El cono sur del estado de Yucatán muestra una variedad en la configuración del relieve, de sierra, lomeríos, cuencas endorreicas y planicies de diversa génesis y formas. En la figura 2, se observa la distribución de las unidades geomorfológicas, destacando $16.3 \%$ del territorio susceptible de inundación, del cual $1 \mathrm{I} .5 \%$ corresponde a inundaciones extraordinarias (depresiones intermontanas (aguadas), conos de eyección y barrancos) y $4.8 \%$ a inundaciones ordinarias (planicies, cenotes, talwegs, terrazas lacustres, zonas de drenaje deficiente, aguadas). En total 73929.9 ha son susceptibles de cualesquiera de estos dos tipos de inundaciones.

\section{Localidades en pobreza extrema}

El estudio de las condiciones socioeconómicas de las localidades del sur de Yucatán se fundamenta en la medición de las Necesidades básicas insatisfechas (NBI) y la Línea de pobreza (LP). Las localidades se diferenciaron en rangos de pobreza extrema que las hace altamente vulnerables a fenómenos naturales desastrosos. El indicador clave es de 0.75 (siendo entre 0.75 y I la pobreza absoluta) que indica el mínimo de elementos materiales para vivir en la sociedad mexicana. 
Áreas susceptibles de riesgo en

localidades de pobreza extrema

96
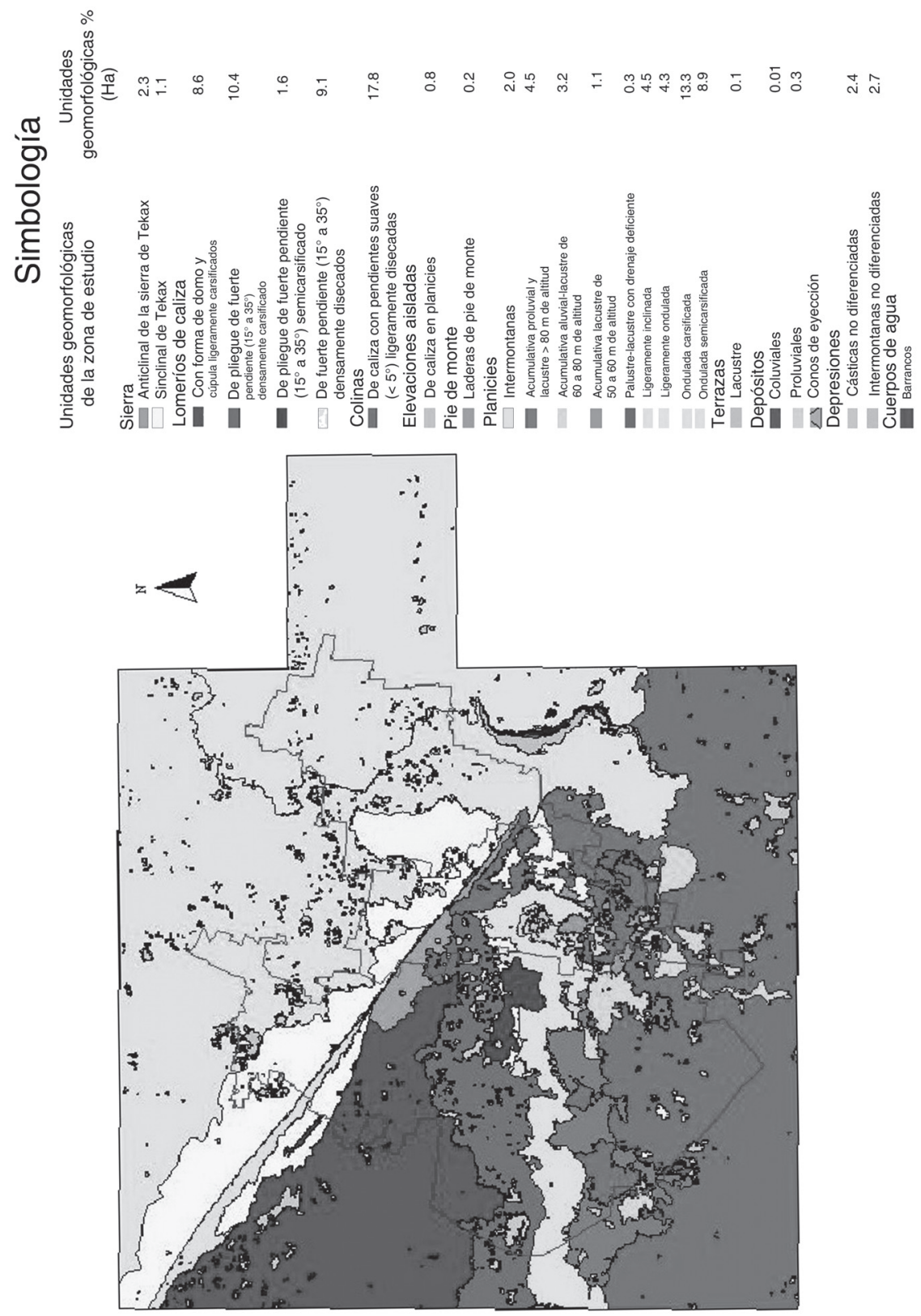

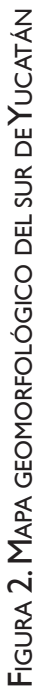



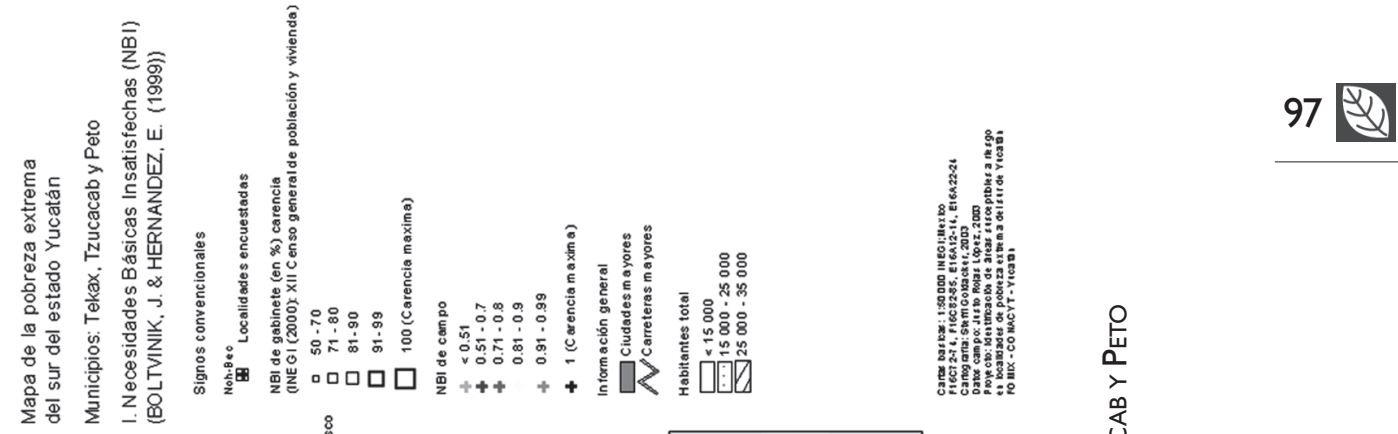

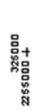
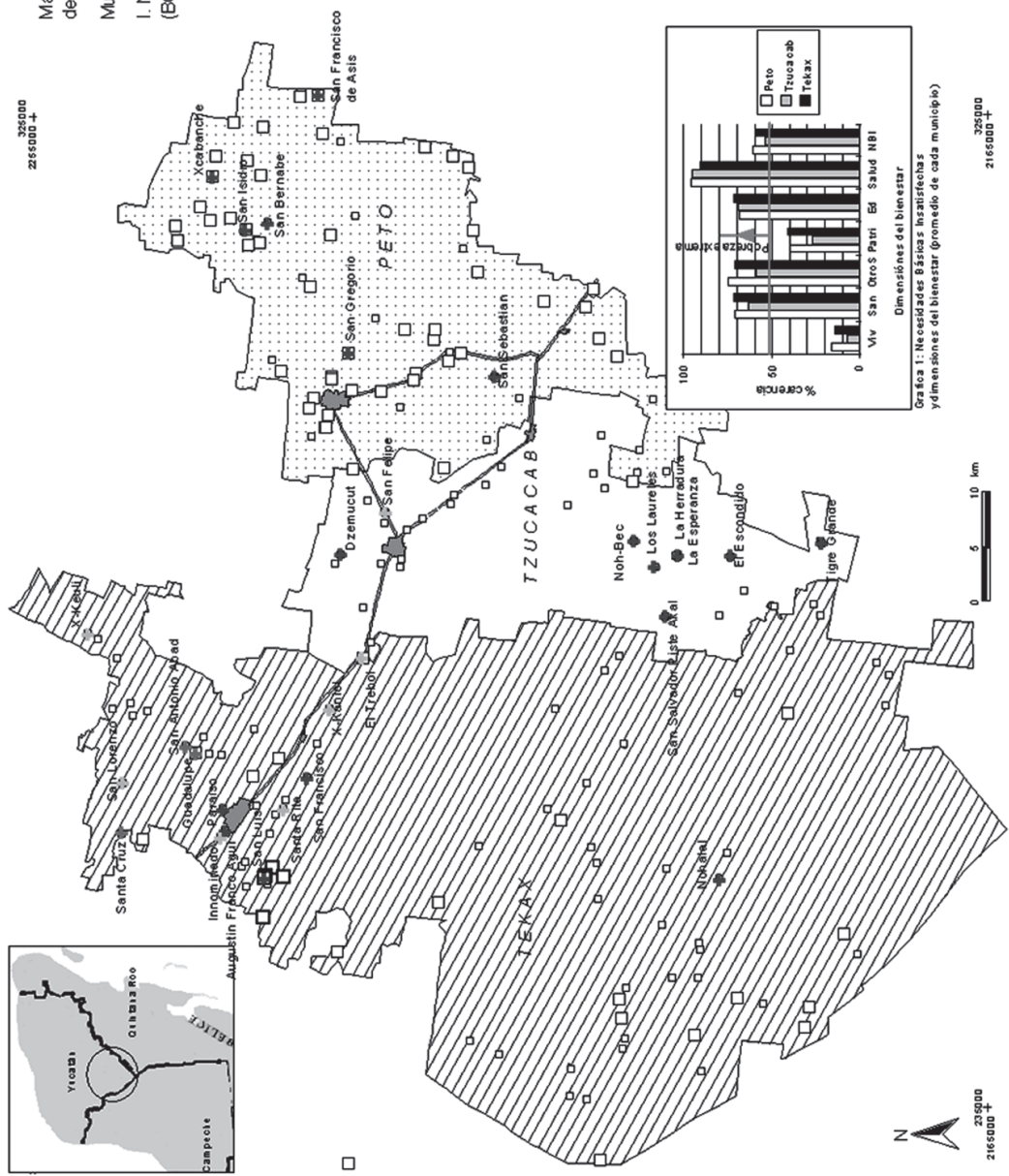

Teoría y Praxis

2 (2006: 87-I03) 
En la figura 3 se muestra la distribución de las localidades en pobreza extrema del sur de Yucatán. Así, tres localidades que fueron afectadas por las inundaciones de 2002 sirven de ejemplo para "calibrar" el modelo de pobreza extrema:Tigre Grande (municipio de Tzucacab) registra un índice de NBI de 0.63 y se clasifica como localidad en pobreza extrema. La localidad de Uitzina (Peto) registra 0.68 y Dzuzal Chico en Tekax tiene el índice de 0.70, ambas localidades en pobreza extrema. Estos tres ejemplos revelan perfectamente la situación por la que atraviesa el sur de Yucatán.

Cabe destacar que se han trabajado con datos de 2000 (en gabinete) y 2003 (en campo), pero se reconoce que a partir de las inundaciones de 2002 las condiciones socioeconómicas de NBI y LP marcan un avance significativo hacia la pobreza absoluta. En total, 187 localidades pequeñas sufren deficiencia social y económica. Por lo tanto, son altamente vulnerables a desastre.

\section{Localidades en riesgo de inundaciones en el sur de Yucatán}

Una vez concluidos los análisis sectoriales entre la susceptibilidad del relieve y de las localidades, se logra la identificación de las localidades en pobreza extrema susceptibles de riesgo de inundaciones (figura 4); así, se tiene:

a) 187 localidades en extrema pobreza

b) 56 localidades en áreas susceptibles de inundación

c) I3I localidades ubicadas en zonas no susceptibles de inundación

En sí, 30\% de las localidades del sur de Yucatán se encuentran en áreas susceptibles de riesgo de inundación. De éstas, 26 localidades se localizan en áreas de inundación ordinaria y 30 en áreas de inundación permanente, lo cual representa a más de 600 familias.

Finalmente, las localidades en pobreza extrema en riesgo de inundación son:

San Salvador y Pisté Akal;Tigre Grande; Blanca Flor; La Esperanza; Huntura Dos; San Manuel; Benito Juárez; La Herradura; Telil; Nueva Santa Cruz; Mesatunich; Mac-Yan; Huntochac; San Diego B., San Felipe Segundo; San Juan T., San Martín Hili; San Pedro Azula; Sudzal Chico; San Jorge; Lázaro Cárdenas; Ayim Dos; Cruz Akal; San Gregorio; San Nicolás; Santa Rita; Xcunya; X-kanlol; El Huiro Dos; Chun Wuitz. 


\section{Discusión}

El sur del estado de Yucatán fue seleccionado para crear un modelo de identificación de zonas de riesgo a desastres naturales, en razón de la gran diversidad de unidades y de formas geomorfológicas de su relieve, y por la amplia brecha que existe en las condiciones socioeconómicas de su población, con más de $88 \%$ en pobreza extrema; elementos que no han sido tratados en la literatura especializada sobre riesgo y desastre para la región (véase Lugo y García, 1999 u Oropeza, 1999).

Los análisis presentados, de tipo ambiental y social, demarcan los factores que influyeron ampliamente en el impacto que tuvo el huracán Isidoro en el año 2002. Este modelo ayuda a entender procesos diversos de inundación, de torrentes, de procesos gravitacionales, entre otros. El ejemplo que se presenta es sólo una muestra del efecto semilla que tienen los dos apartados centrales de la investigación (el estudio del relieve y el de las condiciones de pobreza extrema de la población).

El conocimiento de la ubicación y las características (origen, tipo, intensidad, frecuencia y distribución) de las áreas susceptibles de inundaciones permite la planeación y la política regional de estas áreas para elaborar acciones de mitigación, protección y administración, que pueden ser realizadas por las autoridades locales con la colaboración del área de Protección Civil del estado.

La base de datos generada tiene potencial para ser utilizada en el ordenamiento territorial de la entidad. Los mapas de riesgo a escala 1:50 000 realizados para el sur de Yucatán, y su uso a nivel local, deben ayudar a reducir el peligro de la inundación. Los mapas de riesgo permitirán tomar las decisiones para las acciones de mitigación, protección y manejo del riesgo; tienen, también, un alto potencial no solamente para su uso en Yucatán, sino en otras regiones con características físicas, económicas y sociales similares.

Con estos mapas de riesgo, a escala 1:50 000 del cono sur de Yucatán, y la capacitación de usuarios locales (directores municipales, de protección civil, autoridades de planeación y tomadores de decisiones en tiempos de crisis), se puede reducir el riesgo de inundaciones, al proporcionar criterios para la toma de decisiones oportunas.

Asimismo, debido al "efecto semilla" de la base de datos de los mapas geomorfológico y de pobreza, es posible la diferenciación por fenómenos 
Áreas susceptibles de riesgo en

localidades de pobreza extrema

100

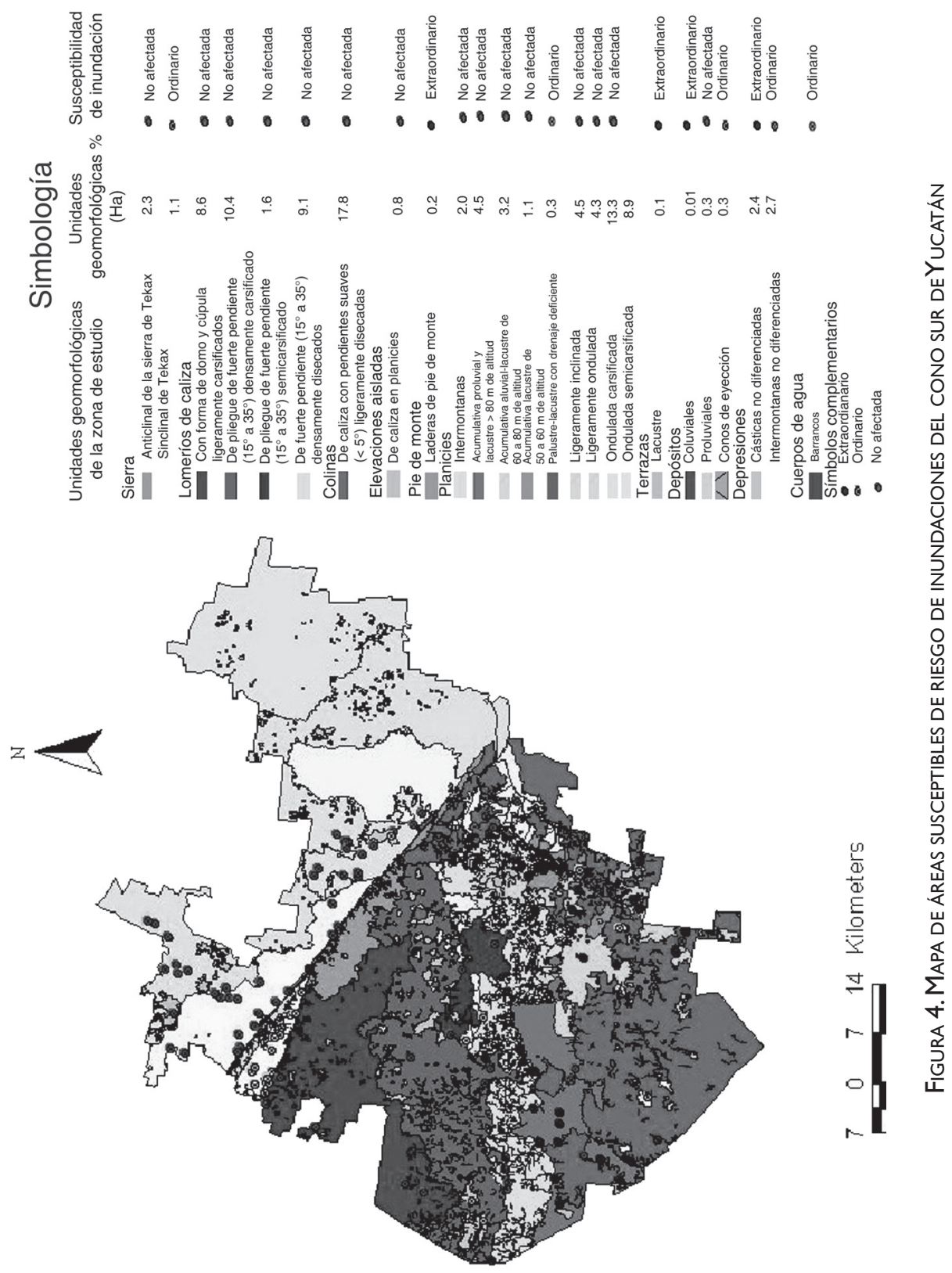


peligrosos (agrietamientos, subsidencias, erosión, derrumbes, hundimientos, etcétera; así como, en calidad de la vivienda, educación, ingresos, entre otros).

\section{Conclusiones}

La identificación de las unidades geomorfológicas, con énfasis en las formas y los elementos del relieve, así como su dinámica ambiental permite diferenciar cuáles son las áreas susceptibles de inundaciones. Es a través de un análisis morfométrico del relieve, de interpretación de fotografías aéreas e imágenes de satélite, además del uso de sistemas de información geográfica, que se logra una cartografía detallada a escala I:50 000.

Conjuntamente, en el análisis de los elementos sociales de cada localidad, destacando aquellas en pobreza extrema y los diversos indicadores socioeconómicos, señala las vulnerables ante fenómenos naturales desastrosos.

Por lo tanto, la correlación de los aspectos ambientales peligrosos y la vulnerabilidad social permitió comprobar la hipótesis de identificación de zonas de riesgo, centrándose en la susceptibilidad de inundaciones durante fenómenos naturales extraordinarios.

La identificación parte de dos aspectos. Primero, el mapeo de las formas y elementos del relieve, que definen la geomorfología; ésta se determina por el análisis del relieve, enfocando la génesis, el proceso y la forma. De ellas se reconocen aquellas áreas susceptibles de desarrollar procesos de inundación, diferenciadas en ordinarias, extraordinarias y permanentes. Conjuntamente, la identificación de localidades en pobreza extrema, tomando en consideración nueve dimensiones socioeconómicas, que se resumen en dos índices (Necesidades básicas insatisfechas y Línea de pobreza). Esta es la hipótesis de la investigación y cuyo objetivo se sintetiza en la presentación del Mapa de riesgo a inundaciones del sur de Yucatán a escala l:50 000 y la Identificación de las localidades en pobreza extrema susceptibles de inundación.

\section{Agradecimientos}

Los autores agradecen el apoyo del Fondo Mixto Conacyt/Gobierno del Estado de Yucatán, número YUC-2002-C0I-8703; a la División de Desarrollo Sustentable de la Unidad Académica Cozumel, Universidad de Quintana Roo; a los presidentes 
municipales de Peto, Tekax y Tzucacab, por el apoyo brindado y a los pobladores de las localidades que nos proporcionaron la información sobre sus necesidades básicas insatisfechas y de la línea de pobreza. A la CNA-Becanchen, CNA Mérida; INEGI, Mérida, CIG de la UQROO, CICY, CINVESTAV, UADY (Dr. Francisco Bautista) por facilitar el acceso a información clave. Finalmente, se agradece la lectura, observaciones y recomendaciones de los dictaminadores, lo cual favorece ampliamente la mejora del manuscrito.

\section{FUENTES CONSULTADAS}

Batista y Sánchez (1995). Clasificación de desastres naturales en Latinoamérica. La Habana: Instituto de Geografía de la Academia de Ciencias de Cuba / Editorial Academia-Cuba.

Boltvinik, J. y E. Hernández (1999). Pobreza y distribución del ingreso en México. México: Siglo XXI.

Burton, I. (1978). The environment as hazards. Nueva York: Oxford University Press.

Burton, I. y R. Kates (1962). "The perception of natural hazards in resource management” Natural Resources Journal, 3, (3), 4I 2-44I.

Burton, I., R. Kates y R. Snead (1969). The human ecology of coastal flood hazard in megalopolis. Chicago: University of Chicago Press.

Cervera, M. y R. Méndez (1999). "Panorama epidemiológico de la península de Yucatán", en Atlas de procesos territoriales del estado de Yucatán. Mérida: Facultad de Arquitectura, UADY, 67-74.

Cordero, M. (1992). El origen y evolución del relieve en la delegación Álvaro Obregón. Tesis de licenciatura, México: FFYLUNAM, 97 P.

Córdova y Ordóñez, C. (1999). “Población y doblamiento”,en Atlas de procesos territoriales del estado de Yucatán. Mérida: Facultad de Arquitectura, UADY, I 2-26.

Frampton et al. (1997). Hazards classification. John and Wiley.

Frausto, O. (2002). Análisis geomorfológico aplicado a los procesos de ladera en la sierra de Guadalupe, México.Tesis de maestría en Geografía: Facultad de Filosofía y Letras, UNAM, $189 \mathrm{p}$.

Hewitt, K. (1997). Geographical study of disasters. Nueva York:Allen and Unwing. Lugo, H. y M.T. García (1999). “El relieve de la península de Yucatán”, en Atlas de procesos territoriales del estado de Yucatán. Mérida: Facultad de Arquitectura, UADY, 155-162. 
Mittchel, J. (1980). "Confronting natural disaster: an international decade for natural hazards reduction" Environmental, 30, (2), 25-29.

Orellana, R. et al. (1999). "Evaluación climática", en Atlas de procesos territoriales del estado de Yucatán.. Mérida: Facultad de Arquitectura, UADY, 163-182.

Oropeza, O. (1999). "Impacto ambiental y peligros naturales y antrópicos en la Península de Yucatán”, en Atlas de procesos territoriales del estado de Yucatán. Mérida: Facultad de Arquitectura, UADY, 248-258.

Ortiz, P. (1996). Fenómenos peligrosos que provocan desastres en México. Atlas Nacional de México. Hoja V.2.9. México: Instituto de Geografía-UNAM.

Ortiz, P. et al. (1992b). "Susceptibilidad a corrimiento de tierras en la cuenca de México", en Atlas Nacional de México, Hoja V.2.I0. México: Instituto de Geografía- UNAM.

--------- (1997). Mapa de riesgos geomorfológicos de la República Mexicana. Escala I:I 000 000. México: INE / Instituto de Geografía, UNAM.

Palacio, A. G. (1995). "Ensayo metodológico geosistémico para el estudio de los riesgos naturales”. Tesis de maestría. México: Facultad de Filosofía y Letras-UNAM.

Whyte, G. (1975). "Natural hazards research: concepts, methods and policy" en G. Whyte (ed.), Natural Hazards: Local, National, Global. Nueva York: Oxford University Press, 3-16.

Zamorano et al. (1992). "Susceptibilidad a inundaciones en la cuenca de México", en Atlas Nacional de México, hoja, v.2.I0. México: Instituto de Geografía, UNAM. 


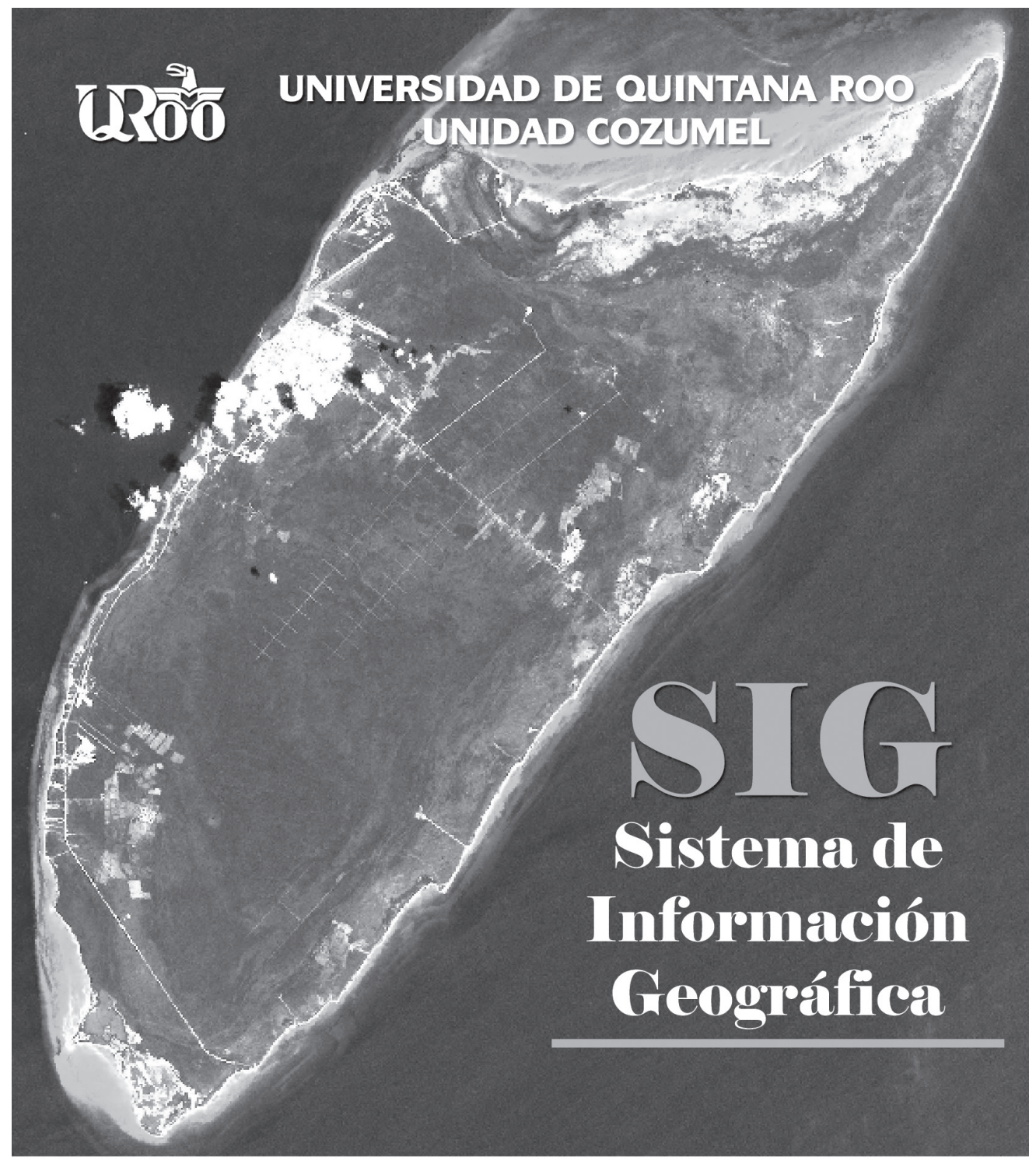

El Sistema de Información Geográfica de la Universidad de Quintana Roo, con apenas tres años de servicios, ha tenido importantes logros en la adquisición y creación de software y hardware.

Entre las ventajas que podría tener este Sistema de Información Geográfica para una comuna como la de Cozumel figura el manejo del Catastro, de la información sobre negocios, de Protección civil, de Desarrollo Urbano, de Protección al Medio ambiente, entre otros grandes rubros. 